
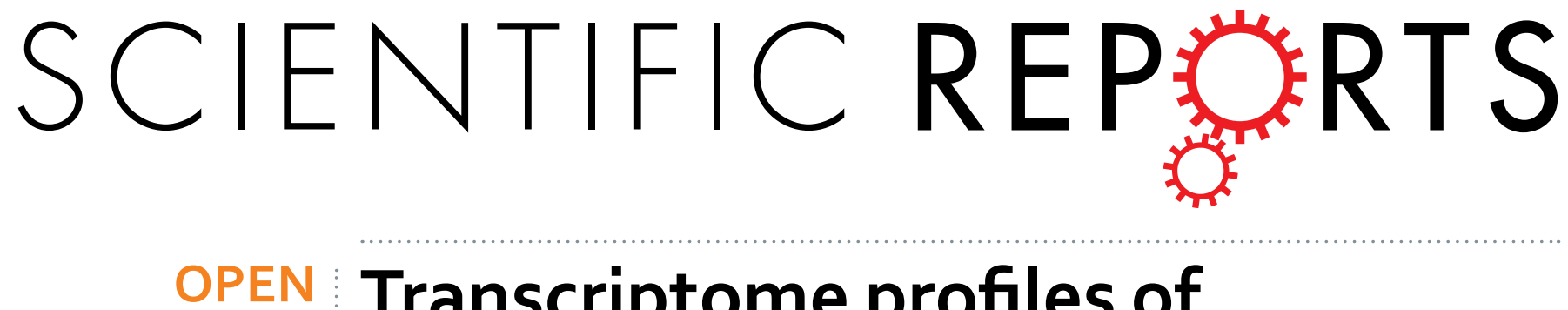

\title{
Transcriptome profiles of
} metamorphosis in the ornamented pygmy frog Microhyla fissipes clarify

Received: 03 February 2016

Accepted: 16 May 2016

Published: 02 June 2016 \section{the functions of thyroid hormone receptors in metamorphosis}

\author{
Lanying Zhao ${ }^{1,2}$, Lusha Liu ${ }^{1}$, Shouhong Wang ${ }^{1,2}$, Hongyuan Wang ${ }^{3}$ \& Jianping Jiang ${ }^{1}$
}

Anuran metamorphosis is an excellent system in which to study postembryonic development. Studies on Xenopus (Mesobatrachia) show that thyroid hormone receptors (TRs) regulate metamorphosis in a ligand-dependent manner by coordinating the action of hundreds of genes. However, whether this mechanism is conserved among amphibians is still unknown. To understand the molecular mechanism of this universal phenomenon, we report the transcriptional profiles of the three key developmental stages in Microhyla fissipes (Neobatrachia): premetamorphosis (PM), metamorphic climax (MC) and completion of metamorphosis (CM). In total, 2,293 differentially expressed genes were identified from comparisons of transcriptomes, and these genes showed stage-specific expression patterns. Unexpectedly, we found that TR $\alpha$ was highly expressed in Xenopus laevis and Bufo gargarizans at premetamorphosis but showed low expression in $M$. fissipes. In contrast, TR $\beta$ was highly expressed during metamorphosis in $M$. fissipes and $X$. laevis. This result may imply that $\operatorname{TR} \beta$ is essential for initiating metamorphosis, at least in $M$. fissipes. Thus, our work not only identifies genes that are likely to be involved in Neobatrachia metamorphosis but also clarifies the roles of unliganded TR $\alpha$ in regulating tadpole growth and timing of metamorphosis, which may be conserved in anurans, and the role of liganded $T R \beta$ in launching metamorphosis.

Metamorphosis is a postembryonic developmental process in which animals reshape and restructure their body's morphology, anatomy and even physiology due to cell proliferation, programmed cell death, cell differentiation, and tissue remodeling ${ }^{1}$. It is an important developmental transition that occurs in many animals, such as some insects, fishes, amphibians, mollusks, crustaceans, cnidarians, echinoderms, and tunicates ${ }^{2-4}$.

Metamorphosis especially in anurans of amphibian is an exquisite model for postembryonic development in vertebrates ${ }^{5-7}$ and thyroid hormones (THs) play essential roles in regulating metamorphosis in anurans. Studies on anuran metamorphosis are mainly conducted in Xenopus (Mesobatrachia). Based on the concentrations of endogenous TH and the morphological characters, Xenopus metamorphosis can be divided into 3 main stages $^{8,9}$ : premetamorphosis (PM), prometamorphosis (POM) and metamorphic climax (MC). In PM, when TH is absent, the unliganded thyroid hormone receptor/9-cis-retinoic acid receptor (TR/RXR) heterodimers recruit corepressors to repress downstream genes and prevent premature metamorphic changes ${ }^{10}$. TH becomes available during POM, and its level throughout the body increases and peaks at the MC stage ${ }^{11}$. Once liganded with $\mathrm{TH}$, the TR/RXR heterodimers recruit coactivators to activate the same downstream genes, leading to metamorphic changes ${ }^{10,12,13}$. This extensive process is mainly characterized by organ resorption, tissue morphogenesis and tissue remodeling ${ }^{8}$. Subsequently, endogenous TH levels drop to a low but measurable concentration at the completion of metamorphosis $(\mathrm{CM})^{14}$. Thus, TRs/RXRs, as switches, regulate Xenopus metamorphosis in a ligand-dependent manner by coordinating the action of hundreds of genes. These genes are associated with many cellular processes, including cell proliferation, programmed cell death and cell differentiation.

${ }^{1}$ Chengdu Institute of Biology, Chinese Academy of Sciences, Chengdu 610041, China. ${ }^{2}$ University of Chinese Academy of Sciences, Beijing 100049, China. ${ }^{3}$ College of Life Sciences, Shaanxi Normal University, Xi'an 710062, China. Correspondence and requests for materials should be addressed to J.J. (email: jiangjp@cib.ac.cn) 


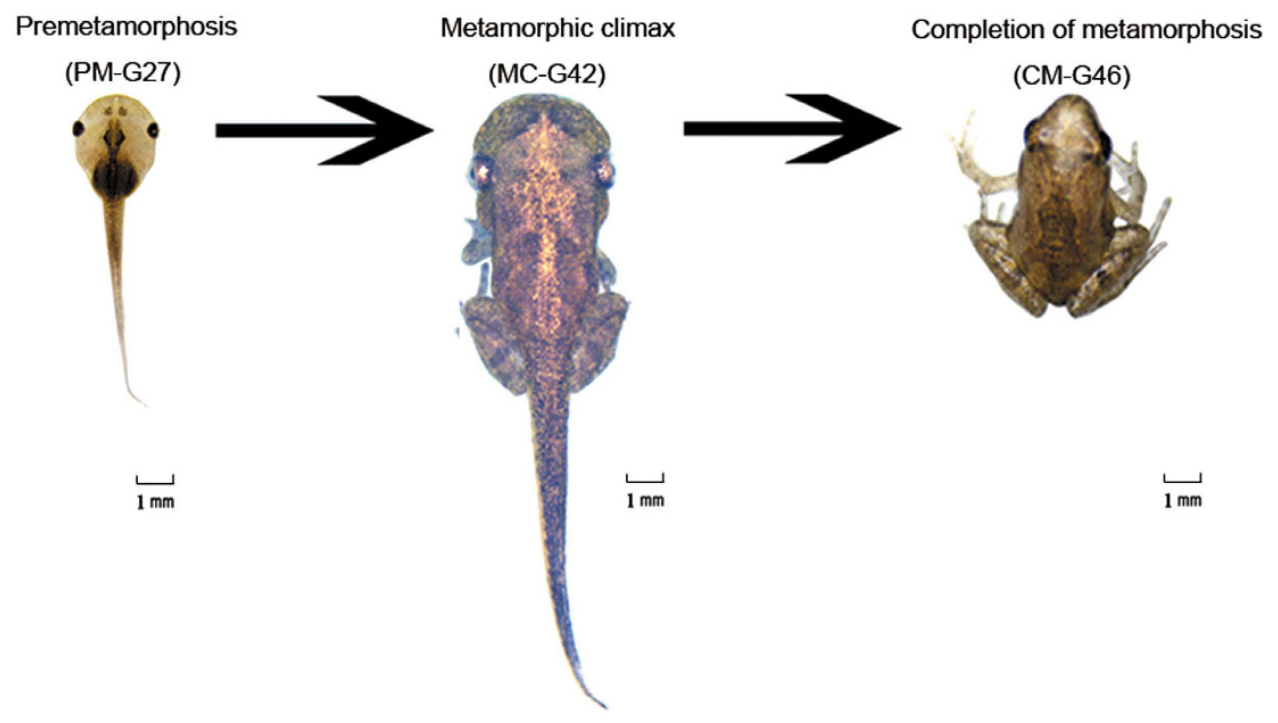

Figure 1. Samples of the three developmental stages used for gene expression profile analysis. Microhyla fissipes in the premetamorphosis (PM), metamorphic climax (MC) and completion of metamorphosis (CM) stages are presented at the left, middle and right, respectively. The scale of the animal's body size is 1:5, and the scale bar is $1 \mathrm{~mm}$.

Xenopus laevis has two TRs, TR $\alpha$ and TR $\beta$, to mediate the effects of TH on the genes that it regulates ${ }^{10,15}$. TR $\alpha$-deficient tadpoles develop faster, and their body size is much smaller than that of their wild-type siblings, suggesting that TR $\alpha$ plays important roles in controlling the timing of metamorphosis ${ }^{16,17}$. Although $\mathrm{TR} \alpha$ has been reported as an essential factor for metamorphosis initiation ${ }^{10,15}$, metamorphosis still occurred in $\operatorname{TR} \alpha$-deficient $X$. laevis. It remains unknown whether $\operatorname{TR} \beta$ or other unknown thyroid hormone receptors are essential for metamorphosis initiation.

Because Xenopus is only a representative of Mesobatrachia, it is necessary to choose another species, ideally one from the Neobatrachia, to investigate whether the molecular mechanism of metamorphosis regulation is conserved in other anuran species. In addition, given the role of TR $\alpha$ in regulating developmental rate and body size in Xenopus, for this study, we chose the Neobatrachian M. fissipes because it has a much smaller body size and shorter timing of metamorphosis than Xenopus ${ }^{18}$. RNA-Seq analysis was performed on $M$. fissipes samples from 3 key developmental stages (PM, MC, and CM), and then the transcriptome profiles were constructed. Comparison of the transcriptomes identified a total of 2,293 differentially expressed genes, which were then clustered using hierarchical clustering. We performed GO enrichment analysis to identify biological processes in the progression of metamorphosis. We also clustered differentially expressed genes using a self-organizing map (SOM), and genes showing stage-specific expression patterns and that may play important roles in specific stages were identified. Notably, the expression level of $\mathrm{TR} \alpha$, which is highly expressed in X. laevis, Rana nigromaculata ${ }^{19}$ and B. gargarizans at premetamorphosis, was expressed at low levels in $M$. fissipes in all three stages. Furthermore, we found that TR $\beta$ was highly expressed at the metamorphic climax stage of $M$. fissipes, as it is in X. laevis ${ }^{15}$, R. nigromaculata ${ }^{19}$, Pelobates cultripes, Scaphiopus couchii and Spea multiplicata ${ }^{20}$. Together with recent work in TR $\alpha$-deficient Xenopus tropicalis, our work not only provides genes that are likely involved in Neobatrachia metamorphosis but also provides further evidence implying that TR $\alpha$ is critical for regulating tadpole growth and the timing of metamorphosis. Meanwhile, our data also imply that the liganded TR $\beta$ may be important for launching metamorphosis.

\section{Results}

De novo transcriptome assembly of Microhyla fissipes and transcriptome annotation. We sequenced the genome-wide transcriptomes of three stages of $M$. fissipes, PM, MC and CM (Fig. 1), and obtained totals of 55,656,754, 59,588,250 and 62,400,210 paired-end raw reads, respectively. All of these Illumina pairedend reads have been submitted to the NCBI short read archive (SRA). The Q30 of the 3 samples was more than $90 \%$. After removal of the adaptor sequences and ambiguous or low-quality reads, we obtained a total of $177,585,214$ ( $94.09 \%$ of the raw reads) clean reads from the three stages. These reads were then de novo assembled, generating 110,061 assembled unigenes which had an average length of $732 \mathrm{bp}$ and an N50 of 1,330 bp (Table 1).

To annotate these assembled unigenes, we next carried out BLASTX searches against the NCBI non-redundant protein database $(\mathrm{Nr})$, the NCBI non-redundant nucleotide database $(\mathrm{Nt})$, the Swiss-Prot and euKaryotic Ortholog Groups (KOG), the Gene Ontology (GO) and the Kyoto Encyclopedia of Genes and Genomes Ortholog (KO) databases. In total, 27,267, 12,905, 23,293, 13,459, 26,692 and 12,134 unigenes were annotated based on these databases, respectively (Table 1). The most unigenes were annotated to the $\mathrm{Nr}$ database. The E-value distribution showed that $60.07 \%$ of the annotated sequences had strong homology (E-value below 1E-50), and the similarity distribution showed that $85.61 \%$ of the annotated sequences had a similarity greater than $60 \%$ (Supplementary Table S1 and Supplementary Fig. S1). In addition, functional classification was also performed 


\begin{tabular}{|l|l|c|}
\hline Sequencing results & Number of total raw reads & $\mathbf{1 8 4 , 8 1 7 , 0 2 4}$ \\
\hline \multirow{5}{*}{ Assembling results } & Number of total clean reads & $177,585,214$ \\
\cline { 2 - 3 } & Number of unigenes & 110,061 \\
\cline { 2 - 3 } & Total length (nt) of total unigenes & $80,600,119$ \\
\cline { 2 - 3 } & Mean length (nt) of total unigenes & 732 \\
\cline { 2 - 3 } & N50 (nt) of total unigenes & 1330 \\
\hline \multirow{5}{*}{ Annotation } & Unigenes with Nr & $27,267(24.77 \%$ of \\
& Unigenes with Nt & 110,061 unigenes) \\
\cline { 2 - 3 } & Unigenes with Swiss-Prot & $23,293(21.16 \%)$ \\
\cline { 2 - 3 } & Unigenes with KOG & $13,459(12.22 \%)$ \\
\cline { 2 - 3 } & Unigenes with PFAM & $25,035(22.74 \%)$ \\
\cline { 2 - 3 } & Unigenes with GO & $26,692(24.25 \%)$ \\
\cline { 2 - 3 } & Unigenes with KEGG & $12,134(11.02 \%)$ \\
\cline { 2 - 3 } & Total unigenes annotated & $34,938(31.74 \%$ of \\
& & 110,061 unigenes) \\
\hline
\end{tabular}

Table 1. Statistics for RNA-Seq based sequencing, assembly and functional annotation for Microhyla fissipes.

against the Gene Ontology (GO) and Kyoto Encyclopedia of Genes and Genomes (KEGG) databases. Based on the GO database, 26,692 unigenes were assigned to 51 level-2 GO terms, which were summarized under three main GO categories, cellular component, molecular function and biological process (Supplementary Fig. S2). Within the GO categories cellular component, molecular function and biological process, 17, 12 and 22 level-2 categories, respectively, were identified. KEGG was used to identify potential biological pathways, and 12,134 unigenes were assigned to $268 \mathrm{KEGG}$ pathways (Supplementary Fig. S3). In total, 34,938 unigenes (31.74\% of the 110,061 unigenes) were annotated in at least one database.

Protein coding sequence (CDS) prediction. To extract coding regions from the assembled unigenes, unigenes were first aligned to protein databases in the priority order NR and then Swiss-Prot. Unigenes aligned to a high-priority database were not aligned to a database of lower priority. The process ended when all alignments had been performed. The correct reading frame of the nucleotide sequences of unigenes $\left(5^{\prime}-3^{\prime}\right.$ direction) was defined by the highest rank in the BLAST results, and the corresponding protein sequences were obtained from the standard codon table. In total, 26,323 unigenes were found to contain a CDS. The size-frequency distributions of the CDS and proteins of these unigenes are shown in Supplementary Fig. S4. Unigenes that could not be aligned to any database were scanned with ESTScan ${ }^{21}$ to predict possible CDS. We obtained 51,506 CDS in 51,420 unigenes. The majority of the unigene CDS (92.72\%) assigned by ESTScan were shorter than $500 \mathrm{bp}$ (Supplementary Fig. S4). The protein sequences were obtained from ESTScan as above (Supplementary Fig. S4). In total, 77,743 unigenes were potential protein-coding genes, comprising $70.636 \%$ of the total unigenes.

Global outlook on the gene expression levels during Microhyla fissipes metamorphosis. To obtain a perspective on global gene expression levels in the three key developmental stages of metamorphosis, we performed pairwise comparisons (MC vs. PM, CM vs. MC, and CM vs. PM). In all, 1,181 and 1,400 unigenes (Fig. 2a,b) were significantly up- and down-regulated, respectively (Supplementary Table S2), and these differentially expressed gene sets were combined into one set. We found 2,293 unigenes that were differentially expressed in the three key developmental stages. Among these unigenes, 50 and 62 unigenes were continuously up-regulated and down-regulated, respectively, from the PM stage to the CM stage. To validate the RNA-Seq results, six genes (col2a1, cola1, myl10, myl2, gngt1 and them4) were randomly selected for further confirmation by real-time PCR. The gene rpl37 was chosen as the internal control because Rpl37 is a ribosomal protein and its expression level is relatively stable ${ }^{22}$, which was supported by our RNA-Seq result. The real-time PCR results of the six chosen genes were consistent with the RNA-Seq expression profiles (Fig. 3).

To identify molecular pathways involved in the transformation from tadpole to froglet, the 2,293 differentially expressed unigenes were grouped into three major clusters (Supplementary Table S3 and Fig. 2c). These clusters contained 919, 628 and 746 unigenes that were expressed mostly in the PM, MC and CM stages, respectively, indicating that these genes played vital roles in the biological processes of different stages.

To understand the biological pathways of the genes in the three identified expression clusters, we used GO enrichment analysis to identify biological process categories that were statistically enriched in each cluster with reference tothe assembled unigenes. The analysis revealed some overlaps in the GO categories (Supplementary Table S4 and Fig. 4). Enrichment of response to oxidative stress (GO:0006979) in cluster 1 and cluster 2 indicates a higher oxygen-binding rate of tadpole hemoglobin than froglet hemoglobin. In cluster 1, regulation of apoptotic process (GO:0042981) was enriched, which is consistent with apoptosis as an early event during metamorphosis ${ }^{23}$. In cluster 2, $18 \mathrm{GO}$ categories were enriched. These categories included proteolysis (GO:0006508), urea cycle (GO:0000050), DNA catabolic process (GO:0006308) and the metabolism of some amino acids, among others. In cluster 3, 15 GO categories were enriched. These included sensory organ development (GO:0007423), regulation of cell migration (GO:0030334), cell motility (GO:0048870), and regulation of embryonic development (GO:0045995). Interestingly, cell-matrix adhesion (GO:0007160) was enriched in clusters 1 and 2, and 

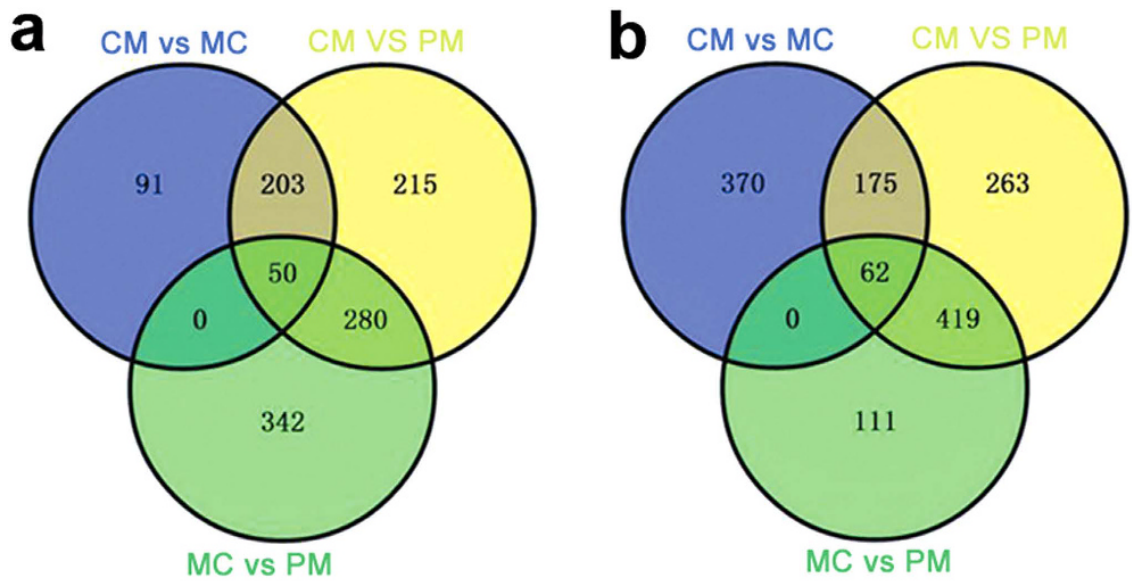

C

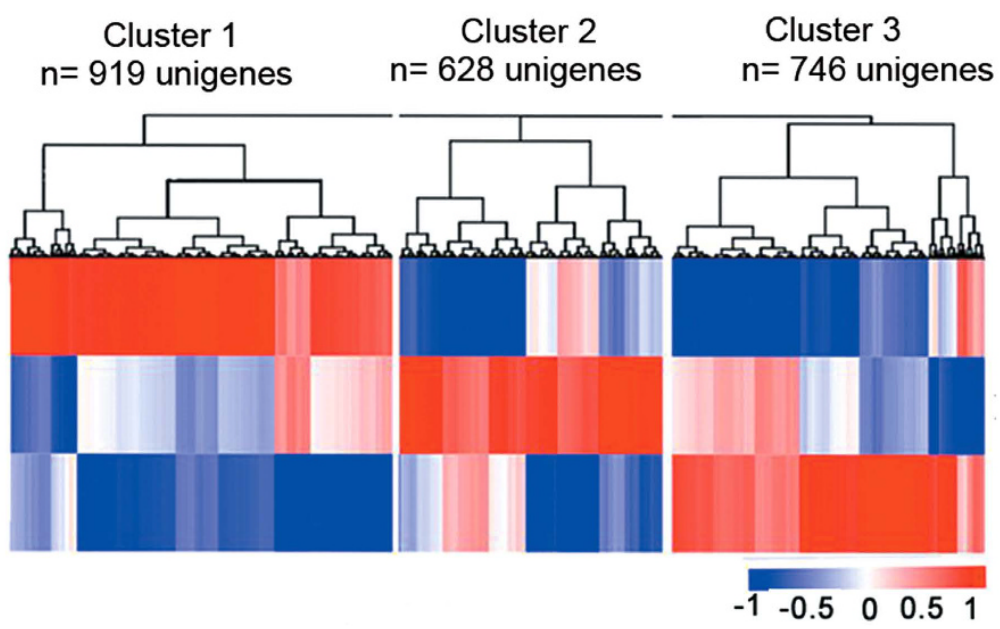

Figure 2. Analysis of differentially expressed unigenes during Microhyla fissipes metamorphosis. Venn diagrams show the number of genes significantly (a) up-regulated and (b) down-regulated; green, blue and yellow circles represent the changes from PM to MC, MC to CM, and PM to CM, respectively. (c) Similarity of the expression profiles between genes with hierarchical clustering is shown above the heatmap. Intensity of color indicates expression levels, and the three major clusters represent the PM, MC and CM stages.

single organismal cell-cell adhesion (GO:0016337) and regulation of cell adhesion (GO:0030155) were enriched in cluster 3. This is consistent with the previous observation that cell-cell and cell-ECM interactions are involved in metamorphosis ${ }^{24,25}$. The modification of the ECM is expected to alter cell-cell and cell-ECM interactions, thus affecting cellular changes including apoptosis, cell proliferation, migration and differentiation (Fig. 4 and Supplementary Table S4).

Stage-specific expression patterns of differentially expressed genes across developmental stages. To further identify genes with roles that vary in importance across developmental stages, expression patterns were deduced according to the fold changes of expression levels in the MC and CM stages compared with that in the PM stage using SOM clustering. In this way, 30 expression patterns were deduced (Fig. 5, Supplementary Table S5). We found 4 clusters that showed stage-specific high expression in one of the three developmental stages (Fig. 5). Unigenes of the 1-3 cluster were specifically expressed in the PM stage. While unigenes of the 4-3 and 5-3 clusters were specifically expressed in the MC stage, unigenes of the 1-6 cluster were specifically expressed in the CM stage. In the PM-specific high-expression cluster (Supplementary Table S6), we found 132 putative stage-specific unigenes, including genes that regulate transcription, such as $c$-myc and $c$-fos. In the MC-specific high-expression cluster (Supplementary Table S7), we observed 122 putative stage-specific unigenes. Genes belonging to the ECM were included, and most of these ECM genes were collagen genes, including the fibrillar forms colla1, col1a2, col3a1, and col5a1 and the non-fibrillar forms col6a1, col6a2, col6a3, and col18a1. In addition, genes related to ECM proteolysis were also included, such as matrix metalloproteinases (MMPs) and a disintegrin and metalloproteinase (ADAM). We also found the hedgehog target gene twist 2 among the MC pattern genes. In the CM-specific high-expression cluster (Supplementary Table S8), we observed 135 putative stage-specific unigenes. GO enrichment analysis at the CM stage showed that a majority of enriched GO categories were associated with metabolic and catabolic processes (Supplementary Table S9). This result is consistent with the fact that the animal starts to feed again at the end of metamorphosis. In addition, we found that the tumor suppressor candidate genes kruppel-like factor $4(\mathrm{klf} 4)$ and $m y o 18 b$ were up-regulated in this cluster. 

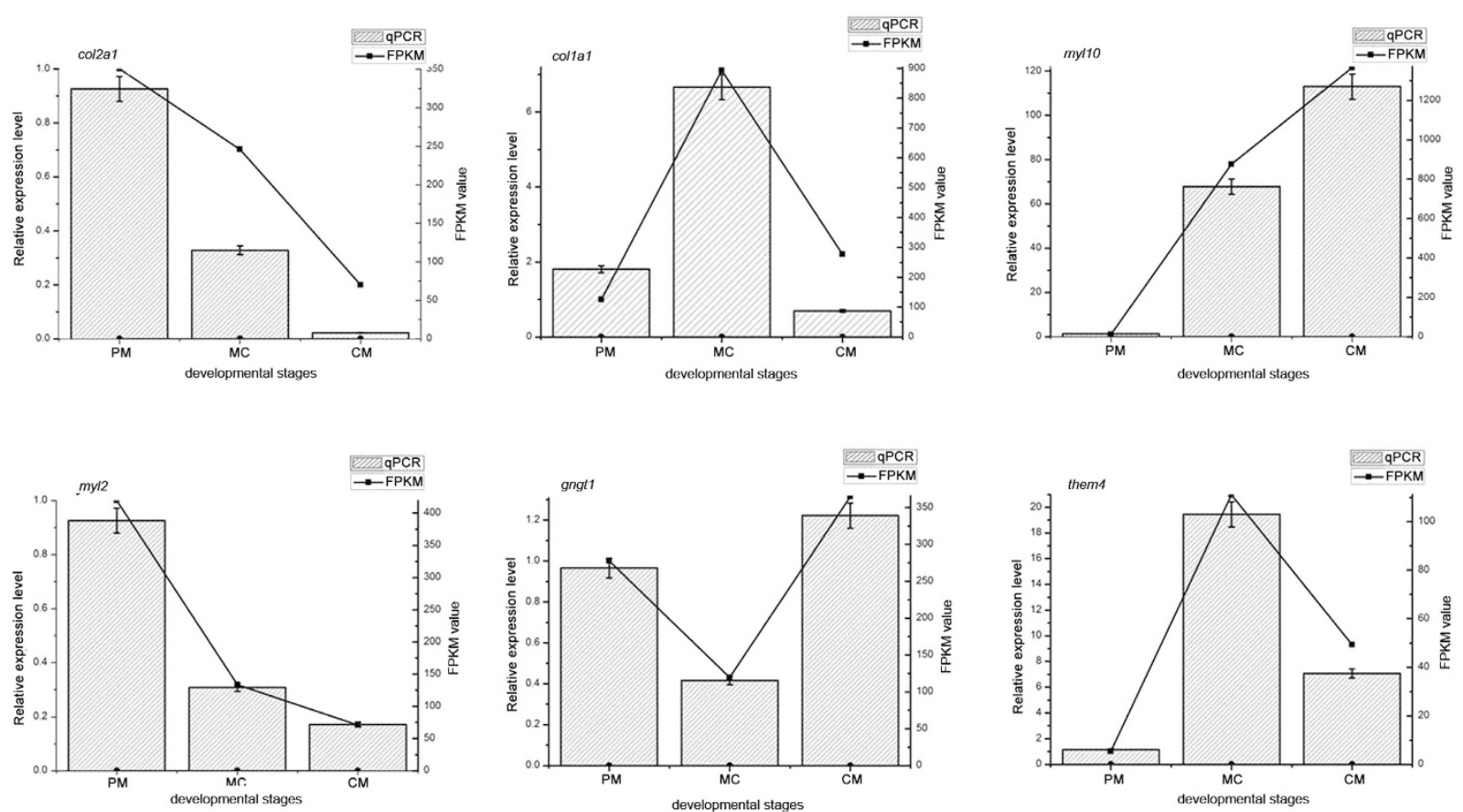

Figure 3. Expression profiles of six genes. The $\mathrm{X}$ axis represents the developmental stages. Lines represent the FPKM value of the transcriptome result (Y axis at right). Columns and bars represent the means and standard errors of three individual samples ( $\mathrm{Y}$ axis at left). Each experiment was performed in triplicate.

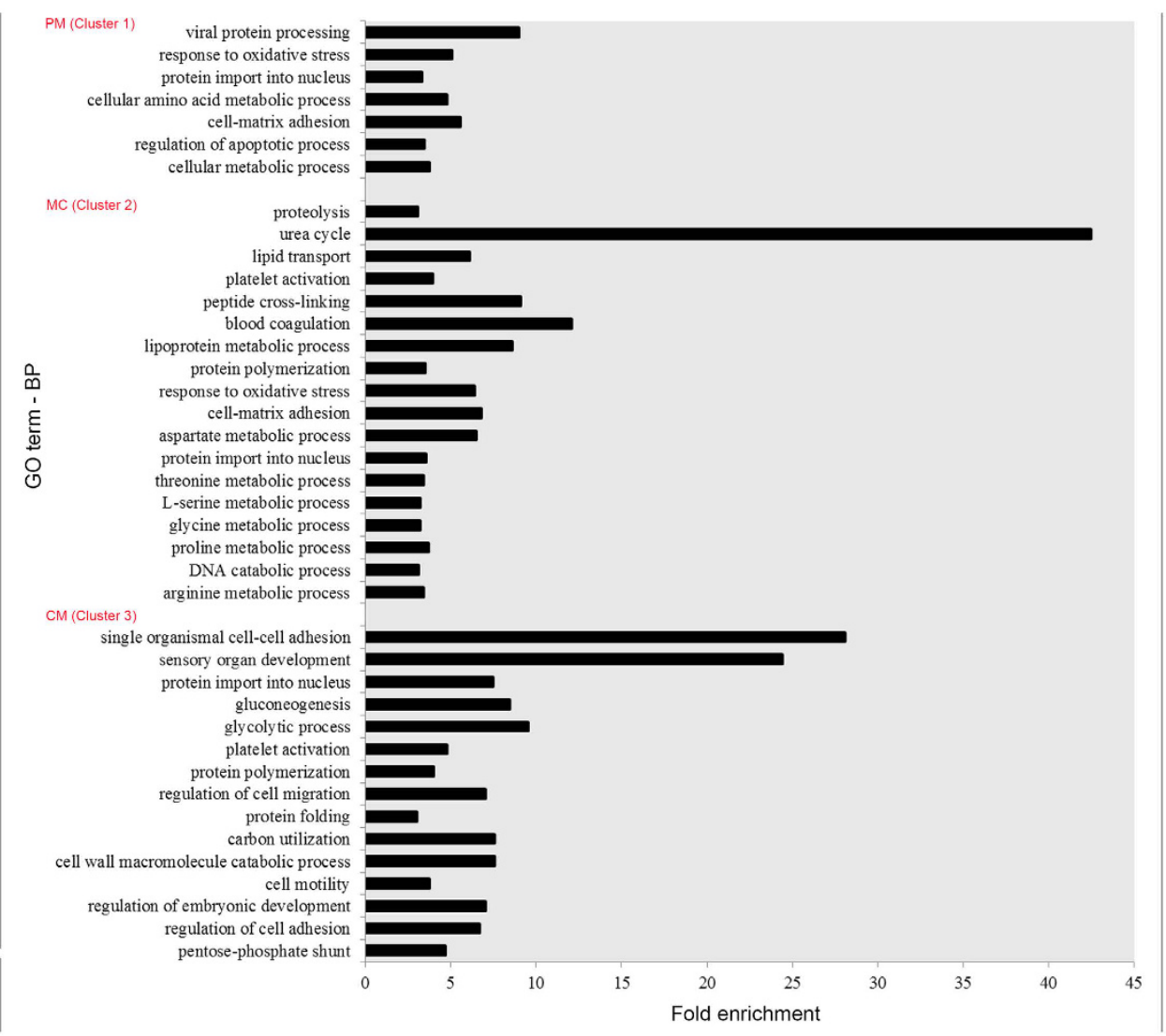

Figure 4. GO enriched processes in three key developmental stages. The significantly overrepresented $($ FDR $<0.05)$ enrichment factors of GO biological processes of the three clusters using hierarchical clustering are shown (Table S7). Only groups containing more than five genes and having a fold enrichment factor $>3$ are presented. 
1-1
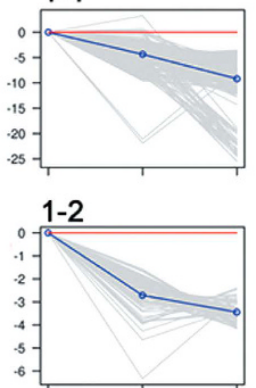

PM

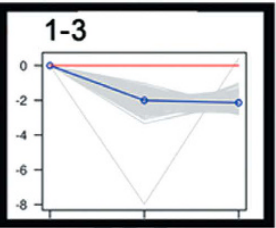

$1-4$

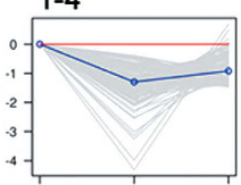

1-5

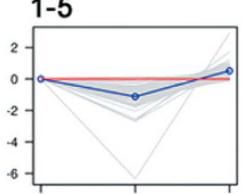

$\mathrm{CM}$

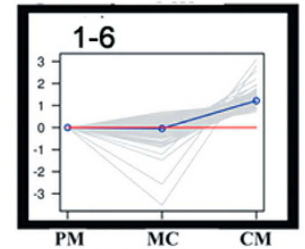

2-1

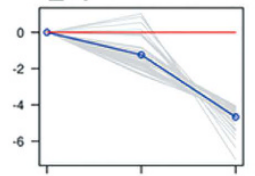

2-2

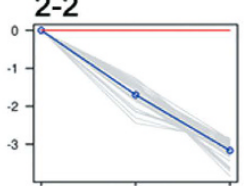

$2-3$

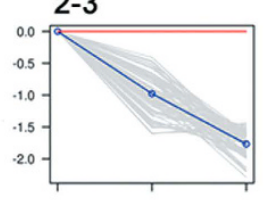

2-4

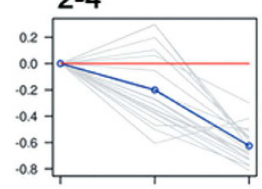

2-5

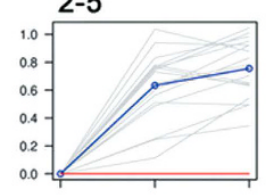

2-6

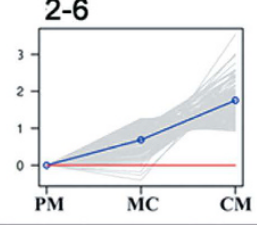

3-1

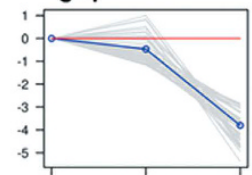

3-2

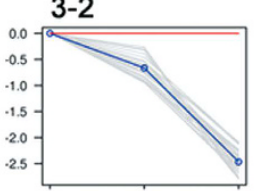

3-3

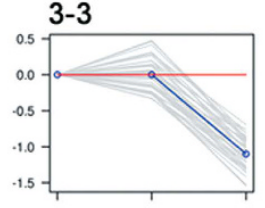

3-4

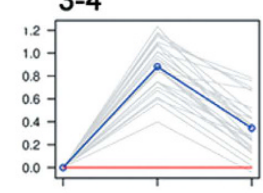

3-5

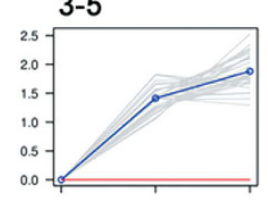

3-6

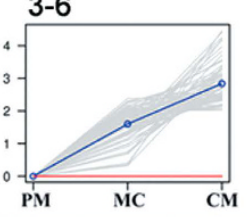

4-1

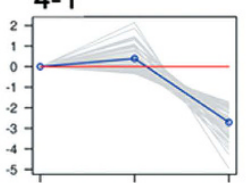

4-2
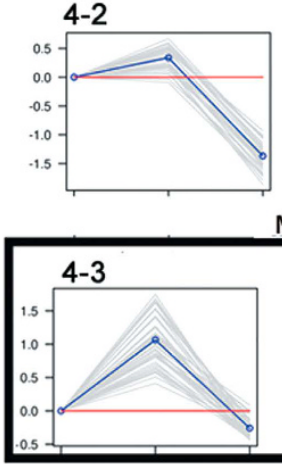

$5-3$

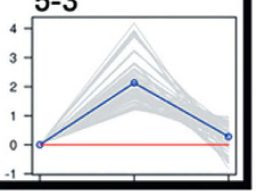

4-4

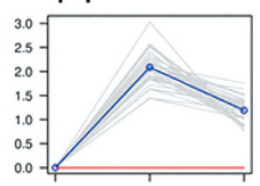

5-4
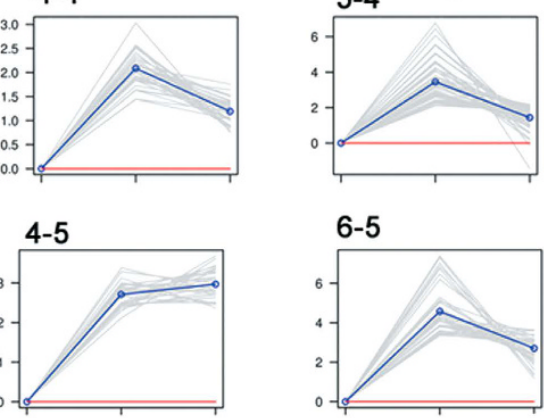

6-5

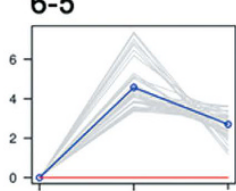

\section{Developmental stages}
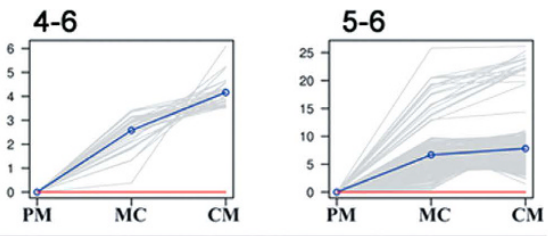

Figure 5. Clustering profiles. Plots of SOM profiles of the reduced set with their centroids highlighted in blue. Four clusters that were further analyzed for stage-specific expression patterns are marked in black boxes. The vertical axis represents the relative gene abundance, and the horizontal axis shows the three developmental stages.

The expression profiles of genes related to the initiation of metamorphosis. The TR/RXR heterodimer plays vital roles in sensing TH and regulating metamorphosis. In all, based on the annotation results, we detected five genes ( $\mathrm{TR} \alpha, \mathrm{TR} \beta, \mathrm{RXR} \alpha, \mathrm{RXR} \beta$, thyroglobulin (TG)) that were involved in initiating the process of metamorphosis. As shown in Fig. 6a, the expression level of $T G$, in parallel with $\operatorname{TR} \beta$ and $\operatorname{RXR} \beta$ mRNA, increased and reached a peak in the MC stage and then declined in the CM stage to the same level as in the PM stage. Interestingly, we noted that the expression level of $\operatorname{TR} \beta$ was higher than that of TR $\alpha$ in $M$. fissipes. To further validate this result, we measured the expression levels of TR $\alpha$ and TR $\beta$ in M. fissipes samples from the three different stages using absolute real-time quantitative PCR. The results showed that the expression level of TR $\beta$ was higher than that of TR $\alpha$ in M. fissipes at all three stages (Fig. 6b). In addition, we also measured the expression levels of TR $\alpha$ and TR $\beta$ in Bufo gargarizans at Gosner stage 27 by relative real-time quantitative PCR (Fig. 6c). The results showed that, unlike in $M$. fissipes, the expression level of TR $\alpha$ was higher than that of $\operatorname{TR} \beta$ at premetamorphosis in X. laevis ${ }^{15}$ and B. gargarizans.

The timing of metamorphosis and the regulation of body length in three anuran species. We also measured the metamorphosis timing and body size of three anuran species (M. fissipes, X. laevis and B. gargarizans) that were reared under identical laboratory conditions $\left(20^{\circ} \mathrm{C}\right)$. It took only 21 days for $M$. fissipes to reach Gosner stage 33 (metamorphosis onset). However, B. gargarizans and X. laevis took 29 and 30 days, 

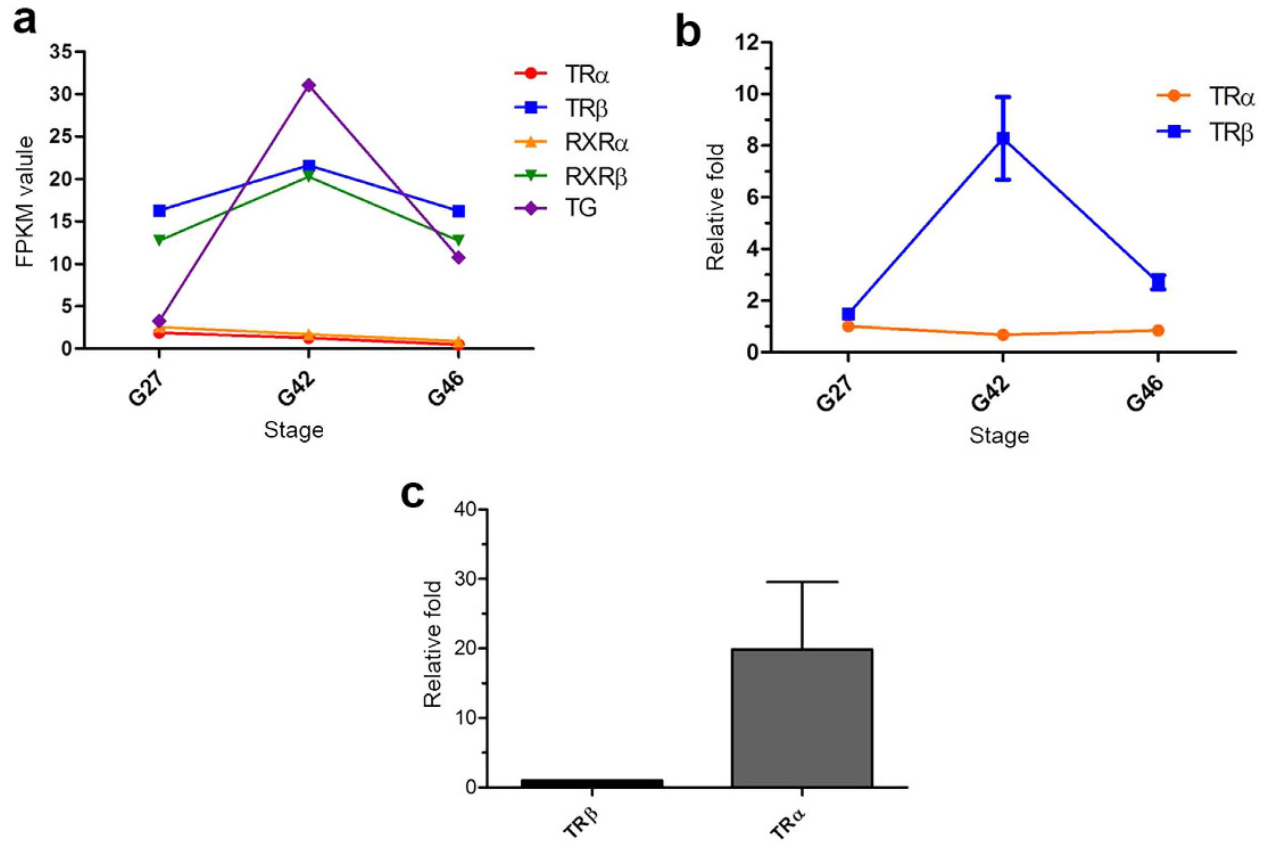

Figure 6. Expressional profiles of thyroid hormone (TH) - related genes involved in regulating metamorphosis. (a) Correlation of the expression levels of $\operatorname{TR} \alpha, \operatorname{RXR} \alpha, \operatorname{TR} \beta, \operatorname{RXR} \beta$ and $T G$ mRNAs with the three key developmental stages of $M$. fissipes. The vertical axis represents expression levels (FPKM value), and the horizontal axis represents developmental stages. (b) Expression profiles of TR genes in three key developmental stages of $M$. fissipes. The X axis represents the developmental stages. Lines and bars represent the means and standard errors of three individual samples ( $\mathrm{Y}$ axis). Each experiment was performed in triplicate. (c) Comparison of the expression levels of TR genes in B. gargarizans at the PM stage. The X axis represents two TR genes. Columns and bars represent the means and standard error of three individual samples. Each experiment was performed in triplicate.

respectively, to attain metamorphosis onset (Supplementary Table S10). Meanwhile, at the onset of metamorphosis, $M$. fissipes had the shortest body length $(17.973 \pm 0.344 \mathrm{~mm})$, with B. gargarizans and X. laevis being longer, at $21.22 \pm 0.384 \mathrm{~mm}$ and $35.956 \pm 0.534 \mathrm{~mm}$, respectively (Supplementary Table S10). Correspondingly, the mean growth rate from stage 19 to stage 33 was $0.735 \mathrm{~mm}$ /day in M. fissipes and $0.592 \mathrm{~mm} /$ day in B. gargarizans, respectively.

\section{Discussion}

Here, we report the transcriptome analysis of $M$. fissipes using the Trinity platform. In this study, comparative expression profiling was performed for the three key developmental stages during $M$. fissipes metamorphosis. This is the first transcriptome analysis spanning the postembryonic process of $M$. fissipes. Our findings not only demonstrate that each stage in the life cycle of $M$. fissipes has a unique expression profile that is involved in particular developmental events but also clarify the functions of TRs (TR $\alpha$ and TR $\beta$ ) in metamorphosis.

PM refers to the stage that includes early tadpole growth and development. The high expression of the proto-oncogenes $c$ - $m y c$ and $c$-fos (Supplementary Table S6) suggests that the larval cells are active in the PM stage. $c$-fos and $c-m y c$ are involved in various processes, including apoptosis and cell proliferation ${ }^{26,27}$. In addition, this result suggests that cell activity is activated in the PM stage, which is consistent with the observation that tadpoles undergo rapid growth in the PM stage ${ }^{8}$. These results provide molecular evidence of the rapid growth of the tadpole body.

Notably, in the MC stage, anurans commonly undergo rapid morphological changes due to the highest endogenous TH levels. Our results show that collagen mRNAs are relatively up-regulated in the MC stage (Supplementary Table S7). Previous studies have found that branching morphogenesis of human mammary epithelial cells is enhanced in collagen I gels ${ }^{28}$. This result indicates that the increasing collagens may accelerate morphogenesis in the MC stage and provides molecular evidence of rapid morphological changes in the MC stage. During MC, most metamorphic changes can be described as profound remodeling of the existing organs, including the liver ${ }^{29}$, skin $^{30}$ and trunk muscle ${ }^{31}$. Tissue remodeling is an important function of matrix metalloproteinases (MMPs) ${ }^{32}$. Among the MMP family members, MMP14 (also known as membrane type 1 MMP; MT1 MMP), MMP19 and TIMP2 were detected in our study. Previous studies showed that MMP14 is associated with TIMP2 at the cell surface ${ }^{33}$ and promotes vascular remodeling ${ }^{34}$. In addition, adamalysin proteins cleave ECM components to influence tissue remodeling ${ }^{35,36}$. Two adamalysin proteins, ADAM19 and ADAMTS7, also showed an MC-specific expression pattern (Supplementary Table S7). Taken together, these results suggest that genes associated with ECM remodeling play a vital role in $M$. fissipes tissue remodeling in the MC stage. In addition, the evolutionarily conserved hedgehog pathway plays very important roles in the process of development. 
A hedgehog target gene twist2, which is reported to promote the epithelial-to-mesenchymal transition (EMT) ${ }^{37}$, is up-regulated in the MC stage, which suggests that the hedgehog pathway participates in the remodeling of the existing organs in the MC stage.

In the CM stage, we found high expression levels of myol $8 \mathrm{~b}$ and $\mathrm{klf4}$, which are associated with negative regulation of the cell cycle (Supplementary Table S8). MYO18B, a class XVIII myosin protein, is a tumor suppressor ${ }^{38}$. In addition, klf4 is a growth arrest-associated gene that inhibits cell proliferation ${ }^{39,40}$. These data suggest that cell proliferation and differentiation are suppressed in the CM stage, which is consistent with the observation that cell proliferation and differentiation are inactive in froglets. Our results provide molecular evidence that the transition from tadpoles to froglets is almost completed in the CM stage.

$\mathrm{TH}$ plays a causative role in amphibian metamorphosis ${ }^{11,41,42}$. It is generally believed that the regulatory function of TH is mediated by TR/RXR heterodimers ${ }^{43,44}$. M. fissipes has two TR (TR $\alpha$ and TR $\left.\beta\right)$ and two RXR (RXR $\alpha$ and $\operatorname{RXR} \beta$ ) genes. We found that in $M$. fissipes, the premetamorphosis expression level of TR $\alpha$ is lower than that of TR $\beta$ (Fig. 6a,b), in contrast to their relative expression levels in Xenopus ${ }^{15}$ and B. gargarizans (Fig. 6c). Meanwhile, $M$. fissipes spends shorter time to attain the onset of metamorphosis and has a smaller body size than either Xenopus or B. gargarizans (Supplementary Table S10). Furthermore, M. fissipes also shows a higher growth rate than that of $B$. gargarizans, which supports the hypothesis that unliganded TR $\alpha$ inhibits growth rate in both M. fissipes and B. gargarizans. These results are consistent with early studies showing that unliganded TR $\alpha$ is expected to repress metamorphic genes ${ }^{45,46}$. Species with low expression of TR $\alpha$ before TH becomes available could show less repression of metamorphic genes and a faster onset of metamorphosis, as seen in TR $\alpha$-mutant experiments in Xenopus ${ }^{16,17}$. TR $\alpha$-deficient tadpoles develop faster and are much smaller in body size than their wild-type siblings. Body size and the timing of metamorphosis are determined by both genetic and environmental factors. Animals in our study were reared under laboratory conditions, which can eliminate the environmental effects. Thus, the fast development and small body size in $M$. fissipes are attributed to the effects of the low expression of TR $\alpha$. It seems clear that TR $\alpha$ plays a role in determining the growth rate and the body size. Our study further underscores the importance of TR $\alpha$ in anuran metamorphosis. In addition, TR $\alpha$ knockdown tadpoles have also delayed natural metamorphosis ${ }^{16}$. However, in our study, it takes longer time to undergo the period of prometamorphosis in M. fissipes than in B. gargarizans and X. laevis. These data could not lead to a conclusion that the liganded TR $\alpha$ has function in accelerating development in M. fissipes as it in X. laevis.

It is worth noting that although the expression level of TR $\alpha$ is very low in all three developmental stages in M. fissipes (Fig. 6a,b), the metamorphic changes still take place. This observation raises the interesting speculation that TR $\beta$ is essential for initiating metamorphosis by activating TH-responsive genes. Firstly, recent work showed that metamorphosis still occurred in TR $\alpha$ mutant $X$. tropicalis tadpoles ${ }^{16,17}$. Secondly, our data show that the expression level of TR $\alpha$ is very low during metamorphosis in M. fissipes (Fig. 6a,b). Thirdly, in X. laevis $\underline{s}^{15}$, R. nigromaculata ${ }^{19}$, P. cultripes, $S$. couchii, $S$. multiplicata ${ }^{20}, M$. fissipes and even TR $\alpha$ mutant $X$. tropicalis ${ }^{16,17}$, TR $\beta$ is still highly expressed during metamorphosis. Last but not least, the TH receptor TR $\beta$ is an important regulator of metamorphosis in Xenopus ${ }^{15}$. Thus, our data suggest that $\operatorname{TR} \beta$ is essential for metamorphosis, at least in M. fissipes.

\section{Methods}

Sample preparation. Microhyla fissipes were collected from paddy fields in Chengdu (Sichuan, China; $103^{\circ} 43^{\prime} 35^{\prime \prime} \mathrm{E}, 30^{\circ} 41^{\prime} 28^{\prime \prime} \mathrm{N}, 541.5 \mathrm{~m}$ ) in May 2014. We raised the animals in the laboratory and applied artificial breeding. We obtained a cluster of tadpoles with the same genetic background. Based on the staging method for anurans $s^{47}$ and using morphological characters, samples were collected in Gosner 27, Gosner 42 and Gosner 46 (Fig. 1), representing the three key developmental periods of PM, MC and CM, respectively. We snap froze samples in liquid nitrogen for 2 hours and then stored them at $-80^{\circ} \mathrm{C}$. To minimize biological variance, nine tadpoles were sacrificed, using three individuals from each of the three stages as independent biological replicates. Animal materials were collected legally. The experimental procedures were approved by the Animal Use Ethics Committee of the Chengdu Institute of Biology. All animal collection and use protocols were carried out in accordance with the appropriate guidelines and regulations.

Total RNA of nine samples was extracted separately using Unizol Reagent (Biostar, China) following the manufacturer's instructions. After RNA purification, concentration and integrity were quantified, and equal amounts of total RNA from the three samples from the same stage were pooled for library construction and sequencing.

cDNA library construction and Illumina sequencing. The mRNAs were purified separately from the 3 RNA samples using poly-T oligo-attached magnetic beads. The mRNAs were subsequently fragmented. First-strand cDNA was synthesized using random hexamer primers and M-MuLV Reverse Transcriptase (RNase $\mathrm{H}^{-}$). Second-strand cDNA synthesis was subsequently performed using DNA Polymerase I and RNase H. The remaining overhangs were converted into blunt ends via exonuclease/polymerase activities. After adenylation of the $3^{\prime}$ ends of the DNA fragments, NEBNext Adaptors with a hairpin loop structure were ligated to prepare for hybridization. To preferentially select cDNA fragments of 150-200 bp in length, the library fragments were purified with AMPure XP system (Beckman Coulter, Beverly, USA). Then, $3 \mu$ l of USER Enzyme (NEB, USA) was used with size-selected, adaptor-ligated cDNA at $37^{\circ} \mathrm{C}$ for $15 \mathrm{~min}$ followed by $5 \mathrm{~min}$ at $95^{\circ} \mathrm{C}$ before PCR. Then, PCR was performed with a Phusion High-Fidelity DNA polymerase, universal PCR primers and Index (X) primer. Finally, PCR products were purified (AMPure XP system), and library quality was assessed on the Agilent Bioanalyzer 2100 system. The clustering of the index-coded samples was performed on a cBot Cluster Generation System using the TruSeq PE Cluster Kit v3-cBot-HS (Illumina) according to the manufacturer's instructions. After cluster generation, the library preparations were sequenced on an Illumina HiSeq 2000 platform by NovoGene (Beijing), and paired-end reads were generated. 
De novo transcriptome assembly and transcriptome annotation. The library quality was verified using FastQC (version 0.10.0) software ${ }^{48}$. Clean reads were produced by removing reads containing adapters, reads containing poly- $\mathrm{N}$ and low quality reads from the raw reads. The clean reads of three libraries were assembled using Trinity ${ }^{49}$ as a reference transcriptome. To annotate the assembled genes of $M$. fissipes, Unigenes were first aligned with BLASTX ${ }^{50}$ to the databases NR, NT and Swiss-Prot with an E-value threshold of 1.0E-5 and to KOG with an E-value threshold of 1.0E-3 to predict the function of genes. Using NR annotation, Blast2GO ${ }^{51}$ software was used to obtain GO annotations defined by molecular function, cellular component and biological process ontologies. Pathway assignments were determined based on the KEGG database using BLASTX with an E-value threshold of 1.0E-5.

Differential gene expression analysis and clustering. For each sequenced library, the read counts were adjusted using the edgeR program package through one scaling normalized factor. Gene expression levels for each sample were estimated using RSEM ${ }^{52}$. To obtain a perspective on global gene expression changes during metamorphosis, we performed pairwise comparisons ( $\mathrm{MC}$ vs. $\mathrm{PM}, \mathrm{CM}$ vs. $\mathrm{MC}$, and $\mathrm{CM}$ vs. $\mathrm{PM}$ ) using the DEGseq (2010) R package ${ }^{53}$ for screening differentially expressed genes. Unigenes were considered as differentially expressed at a normalized fold change $> \pm 2$ at $q$ value $<0.005$ after adjustment for the false discovery rate $(\mathrm{FDR})^{54}$. For better visualization, Venn diagrams show the numbers of unigenes that were significantly up- and down-regulated by pairwise comparisons.

To compare the unigene expression levels, each unigene was further normalized by fragments per kilobase of exon model per million mapped reads (FPKM) for the three developmental stages ${ }^{55}$. For heatmap generation, $\log _{10}(\mathrm{FPKM}+1)$ values were used for each tested unigene. In addition, the differentially expressed genes were also used to deduce expression patterns using SOM (Self Organization Map) clustering analysis based on the $\log _{2}$ (ratios) value (ratios: FPKM of MC and CM were divided by PM, which is considered to represent the initial state) ${ }^{56}$.

Quantitative real time PCR. Samples (three stages of M. fissipes (Gosner 27, Gosner 42, Gosner 46) and one stage of B. gargarizans (Gosner 27)) were used for qRT-PCR. Oligonucleotide primers used for qRT-PCR amplification are listed in Supplementary Table S11. All samples were analyzed in triplicate, and the mean values of these triplicate measurements were used for the calculations of the mRNA levels. The expression patterns of six genes (Gene IDs: comp111682_c3, comp93675_c2, comp47713_c0, comp94949_c0, comp105529_c0, comp47657_c0, comp126565_c0) in $M$. fissipes and the expression levels of $\operatorname{TR} \alpha, \operatorname{TR} \beta$ in B. gargarizans were analyzed using qRT-PCR. A HiScriptTM Q RT SuperMix for qPCR (Vazyme, Nanjing, China) was used to synthesize the cDNAs, and real-time quantification was performed using an Applied Biosystems 7300 Real Time PCR System and the AceQTM qPCR SYBR ${ }^{\circledR}$ Green Master Mix (Vazyme, Nanjing, China). PCR was performed using a program of $95^{\circ} \mathrm{C}$ for $5 \mathrm{~min}$, followed by 40 cycles of $95^{\circ} \mathrm{C}$ for $10 \mathrm{~s}$ and $60^{\circ} \mathrm{C}$ for $30 \mathrm{~s}$. Expression levels of genes in M. fissipes were normalized to that of $r$ pl37. The relative expression levels of genes in $M$. fissipes are presented as $2^{-\Delta \Delta C T}$ values because all the genes tested show highly similar amplification efficiency. The linear regression equations and $\mathrm{R}^{2}$ of TR $\alpha$ and TR $\beta$ in B. gargarizans are shown in Supplementary Fig. S5, and The relative expression of TR $\alpha$ and $\operatorname{TR} \beta$ in $B$. gargarizans was obtained as $\mathrm{N}_{0}(\alpha) / \mathrm{N}_{0}(\beta)=\left(1+\mathrm{E}_{(\beta)}\right)^{\mathrm{Ct}(\beta)} /\left(1+\mathrm{E}_{(\alpha)}\right)^{\mathrm{Ct}(\alpha)}$ which was derived from the basic equation $\left(\mathrm{N}_{\mathrm{t}}=\mathrm{N}_{0}(1+\mathrm{E})^{\mathrm{Ct}}\right)$ that describes the exponential amplification of PCR, where Ct is the number of thermocycles, $\mathrm{E}$ is amplification efficiency, $\mathrm{N}_{\mathrm{t}}$ is the number of amplicon molecules and $\mathrm{N}_{0}$ is the initial number of target molecules ${ }^{57}$. To accurately measure the expression levels of TR $\alpha$ and TR $\beta$ in M. fissipes, we performed absolute quantitative real-time PCR. An equal amount of $M$. fissipes cDNA was used as template to specifically amplify TR $\alpha$ or TR $\beta$. Sequences for the PCR primers are as follows: TR $\alpha$ (sense: AGAATCTTAGCGGGCTGGAC; antisense: AAGAGCTCTGTGGGGCACT), TR $\beta$ (sense: TCAGGGTACATCCCGAGCTA; antisense: CCTCAAACACTTCTAAGAACAGTGG). The PCR products were purified using a gel extraction kit (TIANGEN BIOTECH, Beijing). After confirming the PCR products by sequencing, the accurate concentrations of PCR products were measured using the A260 in a NanoDrop ND1000. Molar concentrations of the template DNA were converted by calculating their molecular weight (TR $\alpha, 752,740$; TR $\beta, 688,034)$. To build standard curves, the quantified PCR products were 10-fold serially diluted and were used as qPCR templates. Standard curves were built in Graph Pad Prism software, and the linear regression equations and $\mathrm{R}^{2}$ were generated in Excel (Fig. S6). The real-time PCR amplification efficiency is calculated as Eff. $=\left[10^{(-1 / \text { slope })}\right]-1$. The quantification of $\operatorname{TR} \alpha$ and $\operatorname{TR} \beta$ cDNA was carried out using real-time PCR as described above. The absolute copy number of TR $\alpha$ was obtained as $\mathrm{N} \alpha=2.34^{*} 10^{(\mathrm{Ct}-15.88) /-3.409}$, and the absolute copy number of TR $\beta$ was obtained as $\mathrm{N} \beta=10.61 * 10^{(\mathrm{Ct}-14.37) /-3.502}$. All the obtained copy numbers of TR $\alpha$ and TR $\beta$ in different samples were then normalized to TR $\alpha$ in Gosner stage 27 by using rpl37 as an endogenous control.

GO enrichment analysis. The GO database covers three domains: cellular component, molecular function and biological process (http://geneontology.org/) ${ }^{58}$. The Blast2GO tool was used to perform GO enrichment for unigenes that showed a different expression during three developmental stages. We used the annotation of the assembled unigenes as a background for the contingency table of the Fisher's exact test. Overrepresented GO terms with a false discovery rate $(\mathrm{FDR})<0.05$ were reported. GO terms in the biological process category were filtered to identify the most representative processes. Only groups containing more than five genes and having a fold enrichment factor $>3$ are presented (Fig. 4).

The observation of body length and developmental timing in three anuran species. Animals were reared under identical laboratory conditions. To document the developmental time of each stage during metamorphosis, the timing at which $4 / 5$ of the population of tadpoles changed in terms of external morphological characters was defined as the developmental time of the corresponding stage (Supplementary Table S10). For the description of metamorphosis initiation, when hind limbs begin to undergo toe differentiation, seven tadpoles 
of each species were sampled at random. Each larva was placed in a shallow dish filled with water, cooled until motionless by adding a piece of ice to the water, and measured for the total length (TOL). The growth rate can be estimated by the formula of (body length (stage 33)-body length (stage 19))/(time (stage 33)-time (stage 19)).

\section{References}

1. Just, J. J., Kraus-Just, J. \& Check, D. A. In Metamorphosis (eds. Etkin, W. et al.) 265-326 (Springer, 1981).

2. Laudet, V. The origins and evolution of vertebrate metamorphosis. Curr. Biol. 21, R726-737, doi: 10.1016/j.cub.2011.07.030 (2011).

3. Truman, J. W. \& Riddiford, L. M. The origins of insect metamorphosis. Nature 401, 447-452 (1999).

4. Holstein, T. W. \& Laudet, V. Life-history evolution: at the origins of metamorphosis. Curr. Biol. 24, R159-161 (2014).

5. Tata, J. R. Amphibian metamorphosis as a model for studying the developmental actions of thyroid hormone. Ann. Endocrinol-Paris 59, 433-442 (1998)

6. Tata, J. R. Amphibian metamorphosis as a model for studying the developmental actions of thyroid hormone. Biochimie 81, 359-366 (1999).

7. Tata, J. R. Amphibian metamorphosis as a model for the developmental actions of thyroid hormone. Mol. Cell Endocrinol. 246, $10-20(2006)$.

8. Dodd, M. \& Dodd, J. The biology of metamorphosis (ed. Lofts, B.) 467-599 (Academic press, 1976).

9. Etkin W. Metamorphosis. (ed. Moore, J. A.) 427-468 (Academic press, 1964).

10. Grimaldi, A., Buisine, N., Miller, T., Shi, Y. B. \& Sachs, L. M. Mechanisms of thyroid hormone receptor action during development: lessons from amphibian studies. Biochim. Biophys. Acta. 1830, 3882-3892 (2013).

11. Etkin, W. The mechanisms of anuran metamorphosis. I. Thyroxine concentration and the metamorphic pattern. J. Exp. Zool. 71, 317-340 (1935)

12. Denver, R. J. Neuroendocrinology of amphibian metamorphosis. Curr. Top Dev. Biol. 103, 195-227 (2013).

13. Nakajima, K., Fujimoto, K. \& Yaoita, Y. Regulation of thyroid hormone sensitivity by differential expression of the thyroid hormone receptor during Xenopus metamorphosis. Genes Cells 17, 645-659 (2012).

14. Leloup, J. \& Buscaglia, M. La triiodothyronine: hormone de la metamorphose de’s amphibiens. C R Acad. 284, 2261-3 (1977).

15. Yaoita, Y. \& Brown, D. D. A correlation of thyroid-Hormone receptor gene-expression with amphibian metamorphosis. Gene Dev. 4, 1917-1924 (1990).

16. Wen, L. \& Shi, Y. B. Unliganded thyroid hormone receptor alpha controls developmental timing in Xenopus tropicalis. Endocrinology 156, 721-734 (2015).

17. Choi, J. Y. et al. Unliganded thyroid hormone receptor alpha regulates developmental timing via gene repression in Xenopus tropicalis. Endocrinology 156, 735-744 (2015).

18. Fei, L., Ye, C. Y. \& Jiang, J. P. Colored Atlas of Chinese Amphibians and their Distribution. 1-620 (Sichuan Science and Thechnology Press, 2012).

19. Lou, Q. Q. et al. Molecular characterization and developmental expression patterns of thyroid hormone receptors (TRs) and their responsiveness to TR agonist and antagonist in Rana nigromaculata. J Environ Sci 26, 2084-2094 (2014).

20. Hollar, A. R., Choi, J., Grimm, A. T. \& Buchholz, D. R. Higher thyroid hormone receptor expression correlates with short larval periods in spadefoot toads and increases metamorphic rate. Gen Comp Endocrinol 173, 190-198 (2011).

21. Iseli, C., Jongeneel, C. V. \& Bucher, P. ESTScan: a program for detecting, evaluating, and reconstructing potential coding regions in EST sequences. Proc Int Conf Intell Syst Mol Biol 7, 138-148 (1999).

22. Olsvik, P. A., Softeland, L. \& Lie, K. K. Selection of reference genes for qRT-PCR examination of wild populations of Atlantic cod Gadus morhua. BMC. Res. Notes 1, 47 (2008).

23. Biswajit, D. et al. Gene expression changes at metamorphosis induced by thyroid hormone in Xenopus laevis tadpoles. Dev. Biol. 299, 342-355 (2006)

24. Sashko, D. et al. Role of ECM Remodeling in Thyroid Hormone-Dependent Apoptosis during Anuran Metamorphosis. Ann NY Acad Sci 926, 180-191 (2000).

25. Shi, Y. B., Fu, L. Z., Takashi, H. \& Atsuko, I. O. Regulation of extracellular matrix remodeling and cell fate determination by matrix metalloproteinase stromelysin-3 during thyroid hormone-dependent post-embryonic development. Pharmacol. Therapeut. 116, 391-400 (2007)

26. Zajac-Kaye, M. Myc oncogene: a key component in cell cycle regulation and its implication for lung cancer. Lung Cancer-J Iaslc. 34, S43-S46 (2001)

27. Eferl, R. \& Wagner, E. F. AP-1: a double-edged sword in tumorigenesis. Nat. Rev. Cancer 3, 859-868 (2003).

28. Berdichevsky, F., Alford, D., Dsouza, B. \& Taylorpapadimitriou, J. Branching Morphogenesis of Human Mammary Epithelial-Cells in Collagen Gels. J. Cell Sci. 107, 3557-3568 (1994).

29. Atkinson, B. G., Warkman, A. S. \& Chen, Y. Thyroid hormone induces a reprogramming of gene expression in the liver of premetamorphic Rana catesbeiana tadpoles. Wound Repair Regen. 6, 323-337 (1998).

30. Suzuki, K. et al. Molecular features of thyroid hormone-regulated skin remodeling in Xenopus laevis during metamorphosis. Dev. Growth Differ. 51, 411-427 (2009).

31. Shimizu-Nishikawa, K., Shibota, Y., Takei, A., Kuroda, M. \& Nishikawa, A. Regulation of specific developmental fates of larval- and adult-type muscles during metamorphosis of the frog Xenopus. Dev. Biol. 251, 91-104 (2002).

32. Marzenna, W. \& Anna, P. Cytokines and extracellular matrix remodeling in the central nervous system. NeuroImmune Biol. 6, 167-197 (2008)

33. Zucker, S. et al. Tissue inhibitor of metalloproteinase-2 (TIMP-2) binds to the catalytic domain of the cell surface receptor, membrane type 1 matrix metalloproteinase 1 (MT1-MMP). J. Biol. Chem. 273, 1216-1222 (1998).

34. Deryugina, E. I., Soroceanu, L. \& Strongin, A. Y. Up-regulation of vascular endothelial growth factor by membrane-type 1 matrix metalloproteinase stimulates human glioma xenograft growth and angiogenesis. Cancer Res. 62, 580-588 (2002).

35. Hite, L. A., Shannon, J. D., Bjarnason, J. B. \& Fox, J. W. Sequence of a cDNA clone encoding the zinc metalloproteinase hemorrhagic toxin e from Crotalus atrox: evidence for signal, zymogen, and disintegrin-like structures. Biochemistry 31, 6203-6211 (1992).

36. Kuno, K. et al. Molecular cloning of a gene encoding a new type of metalloproteinase-disintegrin family protein with thrombospondin motifs as an inflammation associated gene. J. Biol. Chem. 272, 556-562 (1997).

37. Katoh, Y. \& Katoh, M. Hedgehog target genes: mechanisms of carcinogenesis induced by aberrant hedgehog signaling activation. Curr. Mol. Med. 9, 873-886 (2009).

38. Nishioka, M. et al. MY018B, a candidate tumor suppressor gene at chromosome 22q12.1, deleted, mutated, and methylated in human lung cancer. Proc. Natl. Acad. Sci. 99, 12269-12274 (2002).

39. Zhao, W. D. et al. Identification of Kruppel-like factor 4 as a potential tumor suppressor gene in colorectal cancer. Oncogene 23, 395-402 (2004)

40. Ghaleb, A. M. et al. Kruppel-like factors 4 and 5: the yin and yang regulators of cellular proliferation. Cell Res. 15, 92-96 (2005).

41. Gudernatsch, J. Feeding experiments on tadpoles. Archiv für Entwicklungsmechanik der Organismen 35, 457-483 (1912).

42. Allen, B. M. The effects of extirpation of the thyroid and pituitary glands upon the limb development of anurans. J. Exp. Zool. 42, 13-30 (1925) 
43. Sachs, L. M. et al. Dual functions of thyroid hormone receptors during Xenopus development. Comp. Biochem. Phys. B. 126, $199-211(2000)$

44. Sachs, L. M. \& Shi, Y. B. Targeted chromatin binding and histone acetylation in vivo by thyroid hormone receptor during amphibian development. Proc. Natl. Acad. Sci. 97, 13138-43 (2000).

45. Sato, Y., Buchholz, D. R., Paul, B. D. \& Shi, Y. B. A role of unliganded thyroid hormone receptor in postembryonic development in Xenopus laevis. Mech Develop. 124, 476-488 (2007).

46. Buchholz, D. R., Hsia, S. V., Fu, L. \& Shi, Y. B. A Dominant-Negative Thyroid Hormone Receptor Blocks Amphibian Metamorphosis by Retaining Corepressors at Target Genes. Mol. Cell. Biol. 23, 6750-6758 (2003).

47. Gosner, K. L. A simplified table for staging anuran embryos and larvae with notes on identification. Herpetologica. 16, 183-190 (1960).

48. Cock, P. J. A., Fields, C. J., Goto, N., Heuer, M. L. \& Rice, P. M. The Sanger FASTQ file format for sequences with quality scores, and the Solexa/Illumina FASTQ variants. Nucleic Acids Res. 38, 1767-1771 (2010).

49. Grabherr, M. G. et al. Full-length transcriptome assembly from RNA-Seq data without a reference genome. Nat. Biotechnol. 29, 644-65 (2011).

50. Altschul, S. F., Gish, W., Miller, W., Myers, E. W. \& Lipman, D. J. Basic local alignment search tool. J. Mol. Biol. 215, $403-410$ (1990).

51. Conesa, A. et al. Blast2GO: a universal tool for annotation, visualization and analysis in functional genomics research. Bioinformatics 21, 3674-3676 (2005).

52. Li, B. \& Dewey, C. N. RSEM: accurate transcript quantification from RNA-Seq data with or without a reference genome. $B M C$ Bioinformatics 12, 323 (2011).

53. Wang, L., Feng, Z., Wang, X., Wang, X. \& Zhang, X. DEGseq: an R package for identifying differentially expressed genes from RNAseq data. Bioinformatics 26, 136-138 (2010).

54. Storey, J. D. \& Tibshirani, R. Statistical significance for genomewide studies. Proc. Natl. Acad. Sci. 100, 9440-9445, doi: 10.1073/ pnas.1530509100 (2003).

55. Trapnell, C. et al. Transcript assembly and quantification by RNA-Seq reveals unannotated transcripts and isoform switching during cell differentiation. Nat. Biotechnol. 28, 511-515 (2010).

56. Tamayo, P. et al. Interpreting patterns of gene expression with self-organizing maps: Methods and application to hematopoietic differentiation. Proc. Natl. Acad. Sci. 96, 2907-2912 (1999).

57. Livak, K. J. \& Schmittgen, T. D. Analysis of relative gene expression data using real-time quantitative PCR and the 2 -Delta Delta CT method. Methods 25, 402-408 (2001).

58. Gene Ontology, C. The Gene Ontology project in 2008. Nucleic Acids Res. 36, D440-444 (2008).

\section{Acknowledgements}

We thank Huang Yan for critical reading of the manuscript. This work was supported by the Chinese Academy of Sciences [KJZD-EW-L13] and the National Natural Science Foundation of China [No. 31471964].

\section{Author Contributions}

Conceived and designed the experiments: Z.L.Y. and J.J.P. Performed the experiments: Z.L.Y., W.S.H. and W.H.Y. Analyzed the data: Z.L.Y. and J.J.P. Wrote the paper: Z.L.Y., L.L.S. and J.J.P.

\section{Additional Information}

Accession codes: Raw sequence reads can be found in the SRA database under BioProject PRJNA295354. This Transcriptome Shotgun Assembly project has been deposited at DDBJ/EMBL/GenBank under the accession GECV00000000. The version described in this paper is the first version, GECV01000000.

Supplementary information accompanies this paper at http://www.nature.com/srep

Competing financial interests: The authors declare no competing financial interests.

How to cite this article: Zhao, L. et al. Transcriptome profiles of metamorphosis in the ornamented pygmy frog Microhyla fissipes clarify the functions of thyroid hormone receptors in metamorphosis. Sci. Rep. 6, 27310; doi: 10.1038/srep27310 (2016).

(c) (i) This work is licensed under a Creative Commons Attribution 4.0 International License. The images or other third party material in this article are included in the article's Creative Commons license, unless indicated otherwise in the credit line; if the material is not included under the Creative Commons license, users will need to obtain permission from the license holder to reproduce the material. To view a copy of this license, visit http://creativecommons.org/licenses/by/4.0/ 